\title{
Impact of photodynamic therapy vs. ultrasonic scaler on gingival health during treatment with orthodontic fixed appliances
}

\begin{tabular}{|r|l|}
\hline Journal: & Lasers in Surgery \& Medicine \\
\hline Manuscript ID & LSM-18-0150.R3 \\
\hline Wiley - Manuscript type: & Clinical Reports \\
\hline Date Submitted by the \\
Author: & n/a \\
\hline Complete List of Authors: & $\begin{array}{l}\text { Abellán, Rosa; Universidad Complutense de Madrid, Estomatología IV } \\
\text { Gómez, Clara; Instituto de Química Física "Rocasolano" CSIC, Química } \\
\text { Láser } \\
\text { Iglesias-Linares, Alejandro; Universidad Complutense de Madrid, } \\
\text { Facultad de Odontología, Estomatología IV } \\
\text { Palma, J. Carlos; Universidad Complutense de Madrid, Estomatología IV }\end{array}$ \\
\hline Key Words: & $\begin{array}{l}\text { Photodynamic therapy, Ultrasonic scaler, Periodontopathogens, } \\
\text { Cytokines, Orthodontic treatment, Gingival inflammation }\end{array}$ \\
\hline
\end{tabular}

\section{SCHOLARONE Manuscripts}


Title: Impact of photodynamic therapy vs. ultrasonic scaler on gingival health during treatment with orthodontic fixed appliances.

Running Title: Similar impact of PDT vs. US on gingival inflammation.

Authors: Rosa Abellán ${ }^{1}$, Clara Gómez ${ }^{2 *}$, Alejandro Iglesias-Linares ${ }^{1}$, Juan Carlos Palma ${ }^{1}$

${ }^{1}$ Complutense University of Madrid, School of Dentistry, Stomatology IV Department, Plaza Ramón y Cajal sn, 28040 Madrid, Spain

${ }^{2}$ Institute of Physical Chemistry Rocasolano, CSIC, Serrano 119, 28006 Madrid, Spain

\author{
Address of authors: \\ Clara Gómez (author for correspondence)*: \\ Institute of Physical Chemistry Rocasolano \\ Spanish National Research Council, CSIC \\ C/ Serrano 119, 28006 Madrid \\ Tel.: +34-91-561-9400 ext.961252 \\ Fax.: +34-91-564-2431 \\ E-mail address: c.gomez@iqfr.csic.es
}

\title{
ACKNOWLEDGEMENTS
}

This research was supported by a grant from the Eugenio Rodríguez Pascual Foundation (Madrid, Spain) and by the Spanish Research Projects MICINN (Ref.: MAT 2017-83856-C3) and (MAT2015-68837-REDT). Authors wish to thank Intra-Lock Iberia Spanish Enterprise for lending his Periowave ${ }^{\circledR}$ laser system.

\section{Competing interests}

The authors declare no competing interests. 


\section{ABSTRACT}

Objectives: Poor oral hygiene during treatment with fixed appliances results in plaque accumulation. The presence of bacteria in the gingival crevice triggers an inflammatory reaction in the gingival tissues. The aim of this study was to compare the impact of two preventive treatments, photodynamic therapy (PDT) and ultrasonic scaler (US), on gingival health in patients under fixed orthodontic treatment.

Methods: Twenty orthodontic patients were randomly allocated to two groups: PDT or US. Each group received seven sessions [days 0, 15, 30, 45, 90 (3-months follow-up), 180 (6-months follow-up), 270 (9months follow-up)] of experimental interventions, and clinical parameters [Plaque index(PI); gingival index(GI); probing depth(PD)], periodontopathogens [Agreggatibacter actinomycetemcomitans; Porphyromonas gingivalis; Prevotella intermedia; Micromonas micros; Fusobacterium nucleatum; Tannerella forsythia; Campylobacter rectus; Eikenella corrodens; Capnocytopaga sp.] and protein markers [IL-1 $\beta ; I L-1 r a ; I L-6 ; I L-10 ; T N F-\alpha ; F G F-2 / F G F$ basic $]$ were monitored at baseline and at 3, 6, and 9 months. ANOVA, Student's t-test with Bonferroni correction and ANOVA with multiple rank test were used to identify differences between groups $(p<0.05)$.

Results: Clinical assessments [PI, GI and PD] yielded no differences $(p>0.05)$ between groups, which showed a major decrease at the start of the trial. Reductions in total colony forming units (log CFU reduction) were observed with both treatments, although to a greater extent in the PDT group, but with no differences between groups $(\mathrm{p}>0.05)$. Similar reductions in log CFU counts of Porphyromonas gingivalis, Prevotella intermedia and Fusobacterium nucleatum were observed in both groups $(\mathrm{p}>0.05)$. The two groups also showed similar trends for inflammatory mediators with decreased levels of IL-1 $\beta$, IL-10 and TNF- $\alpha$, whereas IL-6 and IL-1ra levels remained stable and those of FGF-2 were increased after both interventions, with no differences $(p>0.05)$ between groups.

Conclusion: Both PDT and US methods proved similar effectiveness for the treatment of gingival inflammation induced by fixed orthodontic appliances. 
Keywords: Photodynamic therapy; Ultrasonic scaler; Periodontopathogens; Cytokines; Orthodontic treatment; Gingival inflammation.

\section{INTRODUCTION}

Orthodontic treatment with fixed appliances generally represents a challenge in maintaining proper oral hygiene and consequently for good periodontal health maintenance, especially in younger patients [1]. Fixed orthodontic appliances facilitate the accumulation of plaque and biofilm development, which can very often induce dysbiosis. A shift in the balance of the normally stable resident oral microbiome leads to worsening periodontal conditions, which can be observed clinically and by immunohistochemical examination [2]. Given a context of pathological inflammation, orthodontic treatment can lead to potentially irreversible periodontal alterations at the cellular [3] and tissue level [4]. To date, the gold standard for the prevention of hard and soft tissues diseases during orthodontic treatment, is still the successful removal of plaque by correct brushing paired with periodic prophylactic scaling by dental professionals $[5,6]$.

The pathogenic potential of supra- and subgingival biofilm resides not only in its microbiome composition but also in its resistance to microbial agents and ability to withstand physical dislodgement during common oral hygiene practices, even mechanical ones [7]. In this regard, alternative antimicrobial methods have been described in the past two decades, such as different kinds of laser radiation or photodynamic techniques (PDT) $[8,9]$. In PDT, a photosensitizer (PS) undergoes transitions through different molecular states to enable the excited PS to interact with surrounding molecules. This interaction leads to the generation of cytotoxic species $[9,10]$. The development of antimicrobial resistance is unlikely since, in microbial cells, singlet oxygen and other free radicals induce cytotoxicity by interacting directly with various pathogen cell structures [11]. The ability to promote cytotoxic activity offers a broad spectrum of action against a wide variety of microorganisms and low mutagenic potential in exposed cells [12].

Only two recent reports have studied some of the effects of PDT as oral preventive treatment in patients undergoing orthodontic treatment $[13,14]$. These studies concluded that PDT reduces gingival index (GI) scores [13] and Streptococcus mutans load in saliva [14], which could benefit orthodontically treated patients. 
It is important therefore to study whether the use of PDT therapy as periodontal maintenance during orthodontic treatment can improve the clinical periodontal parameters by reducing total periodontopathogenic bacteria and so modulate the host immune response and cytokine secretion profile.

Based on these considerations, the main objective of this study was to evaluate and compare in vivo the clinical, antimicrobial and immune-inflammatory outcomes of two different methods: photodynamic therapy (PDT) and ultrasonic scaling (US) for recovery of periodontal health during orthodontic treatment.

\section{MATERIAL AND METHODS}

\section{Study design}

This clinical study was granted Ethical Committee approval by the Institutional Ethics Committee (Comite de Ensayos Clínicos del Hospital Universitario San Carlos, Madrid, Spain. 08/30/2016. CODE: 16/364-E) in accordance with the ethical principles of the World Medical Association Declaration of Helsinki (2013) [15]. Parents and/or legal guardians of the patients signed the Informed Consent. Being the patients over 12 years old but under 18 years old, they were asked to sign an adapted Informed Consent if they voluntarily decided to participate in the study.

A double-blinded full-mouth study of nine-months duration was designed (Figure 1). Patients were randomly allocated to two different treatment groups: group 1: Photodynamic therapy (PDT) or group 2: Ultrasonic scaler: (US). The generation of random allocation sequences and assignment of patients to each group was based on patient's code, and performed by staff who were involved neither in treating the patients nor in the research. Participants and those assessing outcomes (clinical, microbiological and protein marker assessments) were blinded after assignment to intervention. Operators were not blinded due to operative restrictions. This study has been designed following CONSORT statement guidelines. The clinical trial has been pre-registered in the European Clinical Trials Database (European Medicines Agency EudraCT//018000465-36).

\section{Timeline and pre-clinical trial preparation}

Two weeks before the experimental assessment of treatment started, all subjects who had been accepted for inclusion in the study received training in oral hygiene techniques and were given the same toothpaste, 
Study sample

Patients from the Orthodontics Master's Program of the UCM (Complutense University of Madrid, Spain) were consecutively invited to participate in the study. Participants were selected according to the following inclusion criteria: 12-18 years old; under fixed orthodontic treatment (between 12-15 months); bleeding on probing $(\mathrm{BOP}) \sim 15 \%$; GI $>1.1$; plaque index $(\mathrm{PI})>1.1$; probing depth $(\mathrm{PD})<3 \mathrm{~mm}$; absence of oral lesions; absence of diagnosed periodontal disease. The exclusion criteria were: smokers or former smokers; antidepressant treatment with Monoamine oxidase inhibitors (MAOIs); glucose-6-phosphate dehydrogenase deficiency; history of previous periodontal treatment; antibiotic or anti-inflammatory therapy received in the 3 months immediately prior to treatment or during treatment; use of antiseptic mouthwash; use of corticosteroid or immunosuppressant drugs; systemic condition that could affect the response of gingival tissues or the ability to perform adequate plaque control (diabetes, immune system disorders, etc.).

After the application of these restrictive inclusion and exclusion criteria [13] and computing loss and exclusion of patients with incompletely recorded histories, the final study population consisted of 20 systemically healthy subjects (6 female, 14 male, mean age $14.6 \pm 1.6$ years old). Patients underwent a non- 
extraction orthodontic treatment with fixed orthodontic appliances for at least 12 months (018" slot, metal brackets, Hilgers prescription (3M Unitek, USA).

\section{$\underline{\text { Experimental interventions }}$}

\section{Group 1: Photodynamic therapy}

The gingival sulcus of assigned patients was filled with photosensitizer solution (3,7-Bis(dimethyl-amino) phenazathionium chloride trihydrate (methylene blue) $0.005 \%(\mathrm{w} / \mathrm{v})$ ), suspended in a balanced solution of phosphate buffered saline using hydroxymethyl cellulose as a mucoadhesive viscosity agent Periowave $^{\mathrm{TM}}$ Treatment Kit, Ondine Biopharma Corporation, Vancouver, Canada). To ensure complete irrigation of the apical portion of each gingival sulcus, $10 \mu \mathrm{L}$ of the solution was applied directly using a 23-gauge Dentsply blunt-end, side-port delivery irrigation needle (York, PA, USA) (Figure 2A). The photosensitizer was left for $3 \mathrm{~min}$, after which any excess was gently rinsed away. For laser photoactivation, a diode laser was used (Periowave $^{\mathrm{TM}}$, Ondine Biopharma Corporation, Vancouver, Canada), operating at a wavelength of $670 \mathrm{~nm}$ and equipped with a flexible fiber optic cable attached to a custom-designed stainless steel autoclavable handpiece. To allow access to the gingival sulcus, the handpiece accommodated a disposable light-diffusing tip that was configured similar to a periodontal probe. The laser radiation was applied by partially inserting the light-diffusing tip into the sulcus, no deeper than probing depth, and gently moving it along the sulcus for no more than 60s per tooth (Figure 2B). Table 1 shows a detailed description of the laser parameters used in this study. During laser application, the patient, the operator and dental assistants wore safety glasses to protect their eyes.

Patients received four sessions of PDT (with a 15-day interval between sessions) then, reminder sessions at 3 , 6 and 9 months, with microbiological and immunological sampling and clinical assessment [total of seven sessions] (Figure 1).

\section{Group 2: Ultrasonic scaler treatment (control group)}

All patients in group two received oral prophylaxis with an ultrasonic scaler. Briefly, adhered plaque was removed with ultrasonic instrumentation (sonic SONICflex 2003L KaVo Dental, Germany) and a universal accessory tip [SONICflex scaler no.5] (Table 1) (Figure 2C). The session finished when an experienced 


\section{Monitoring and assessment timeline}

\section{Clinical assessment}

At the beginning of the study before clinical monitoring took place, the examiner underwent probing depth calibration training. The examiner evaluated five patients, not related to the study, in two separate sessions 48h apart. Calibration was accepted if measurements at baseline and at $48 \mathrm{~h}$ were within 1 millimetre of each other, with a $>90 \%$ confidence level.

After calibration, the blinded operator performed all clinical assessments [probing depth (PD), plaque index (PI) and gingival index (GI)] [16, 17]. These clinical parameters were recorded immediately before the initial administration of group 1 and group 2 interventions and at the start of the 3-, 6- and 9-month follow-up visits (Figure 1).

PD was measured to the nearest millimetre from the gingival margin to the base of the gingival sulcus; recorded probing depth was measured with a manual periodontal probe (UNC 15, Hu-Friedy Co., Chicago, IL, USA) at six sites on each tooth: mesiobuccal, buccal, distobuccal, distolingual, lingual and mesiolingual. PI and GI were calculated according to the criteria described by Silness \& Löe and Löe respectively [16, 17] by exploring the gingival unit at 4 sites: mesio-buccal, mid-buccal, disto-buccal and mid-lingual. PI [16] was categorized as follows: $0=$ no plaque; $1=$ sparse accumulation of plaque deposits visible with magnifying glasses; 2 = moderate accumulation of plaque deposits which can be seen with the naked eye; $3=$ heavy accumulation of soft material filling the niche between the gingival margin and tooth surface. GI [17] was assessed as follows: $0=$ normal gingival, no inflammation, discoloration or bleeding; $1=$ mild inflammation, slight color change, mild alteration of gingival surface, no bleeding on pressure; 2 = moderate inflammation with edema and redness, swelling, bleeding on pressure; 3 = severe inflammation with marked edema and redness, swelling, tendency to spontaneous bleeding, perhaps ulceration.

Subgingival plaque sample collection and microbiological culture 
To prevent contamination by blood, subgingival plaque was always collected before taking clinical measurements at each of the four specified timepoints (start of the clinical trial and at 3, 6 and 9 months) (Figure 1). Plaque samples were obtained from the sulcus with the deepest probing depth in each quadrant, so that four different samples of gingival sulcus were taken from each patient (third molars were excluded to prevent salivary contamination).

Once the site was isolated with cotton rolls (Hartmann N.1), supragingival plaque was removed with a curette (Hu Friedy, Chicago, IL, USA) without touching the marginal gingiva for proper extraction of the sample. The gingival sulcus was dried with an air syringe and samples of subgingival microbiota were collected by inserting 2 sterile paper points N30 (Dentsply) into the deepest part of the periodontal sulcus for 30 seconds, avoiding possible contamination with saliva and blood.

After sampling, sterile paper points from each site were inserted into sterile vials with screw caps containing $1.5 \mathrm{~mL}$ of RTF and transferred to the Microbiology Laboratory at the Dental School, UCM, Madrid.

Microbiological culture analysis was carried out 2 or 3 hours after sampling. Briefly, subgingival plaque samples were vortexed for 30 seconds to achieve a homogeneous suspension. Serial 1/10 dilutions $\left(10^{-0}\right.$, 10-1, $10^{-2}, 10^{-3}, 10^{-4}$ ) of bacterial suspension in PBS medium (phosphate buffered saline) were made before the samples were processed. For this, $0.1 \mathrm{~mL}$ was taken from the vial and dispersed into a tube with $0.9 \mathrm{~mL}$ of phosphate buffered saline (PBS) $\left(10^{-1}\right)$. Next, $0.1 \mathrm{~mL}$ was taken from the tube and added to another one containing $0.9 \mathrm{~mL}$ of PBS $\left(10^{-2}\right)$, and the process was repeated until there were $10^{-4}$ dilutions, as specified. 0.1 $\mathrm{mL}$ volumes of dilutions 0 and 1 were dispensed into a selective culture medium to determine the presence of Agreggatibacter actinomycetemcomitans (Aa), and $0.1 \mathrm{~mL}$ volumes of dilutions 2, 3 and 4 into blood agar medium to determine the presence of Porphyromonas gingivalis (Pg), Prevotella intermedia (Pi), Micromonas micros (Mm), Fusobacterium nucleatum (Fn), Tannerella forsythia (Tf), Campylobacter rectus (Cr), Eikenella corrodens (Ec), Capnocytopaga sp. (Csp).

The Dentaid medium for $\mathrm{Aa}$ was incubated for 2-3 days at $37^{\circ} \mathrm{C}$ in $5 \% \mathrm{CO}_{2}$ candle jars [18]. Morphological identification of $A a$ was carried out 2 days after seeding. The catalase test was used to confirm the presence of $A a$, with the addition of $3 \% \mathrm{H}_{2} \mathrm{O}_{2}$, which only produces bubbles if positive.

Blood agar medium (Oxoid no.2, Oxoid Ltd, Basingstoke, UK) with 5\% horse blood and 5\% hemin and menadione solution was incubated for 7 days at $37^{\circ} \mathrm{C}$ in anaerobic jars with $80 \% \mathrm{~N}_{2}, 10 \% \mathrm{H}_{2}$ and $10 \% \mathrm{CO}_{2}$. 


\section{Gingival crevicular fluid collection and immunology multiplex assay}

Samples of Gingival crevicular fluid (GCF) were taken from every patient at each of the four specified timepoints (start of the clinical trial and at 3,6 and 9 months) (Figure 1) to determine variations in the levels of six cytokines (IL-1 $\beta$, IL-1ra, IL-6, IL-10, TNF- $\alpha$ and FGF-2 (FGF basic) by Luminex assay; Ref: HCYTOMAG-60K-06; MILLIPLEX ${ }^{\circledR}$-MAP-Human Cytokine/Chemokine Panel 1, Merck Millipore, Billerica, MA, USA).

Samples were taken from locations with the greatest PD, near those selected for subgingival plaque samples (and sampling the same location, in each patient, at each follow-up), excluding the third molars. GCF was collected with commercially available paper strips (Periopaper, Oraflow Inc., Plainview, NY, USA), which were inserted into the gingival crevice until mild resistance was felt, then left in place for $30 \mathrm{~s}$. The volume of collected GCF was measured with a Periotron 8000 (Oraflow Inc., Plainview, NY, USA) (previously calibrated using human serum as calibrant and fitting the data with fourth order polynomial regression) [19]. The periopaper strips were then placed in sterile Eppendorf vials with a filter and stored at $-80^{\circ} \mathrm{C}$ until required for analysis. Samples contaminated with blood or saliva were discarded.

Samples were analyzed in duplicate using multiplex map human high sensitivity immunoassays (Merck Millipore, Billerica, MA, USA) using the Luminex-200 analyzer and XY plate handling platform (Luminex Corporation, Oosterhout, Netherlands). For this, $100 \mu \mathrm{L}$ of phosphate buffer $(\mathrm{pH}=7)$ with protease inhibitor (Complete Mini Cocktail tablets, Roche, ref: 11836153 001), 0.05\% PMSF (Phenylmethylsulfonyl fluoride) $(200 \mathrm{mM})$ and $0.5 \%$ of BSA (Bovine Serum albumin) was added to the periopaper. This mixture was incubated at room temperature for 30 minutes. After that, the Eppendorf tubes were centrifuged for 10 minutes at 10,000 rpm and the filters with the periopapers were discarded. Calibration for microsphere classification and reporter readings as well as sheath fluid were also supplied by Luminex Corporation. Data 
were analyzed with xPonent software (Luminex Corporation, Oosterbout, Netherlands) and expressed as level of each inflammatory biomarker/volume of GCF $(\mathrm{pg} / \mu \mathrm{L})$.

\section{Statistical analysis}

The Shapiro-Wilk test was used to check assumptions of normality for the tested variables. Thus a two-factor repeated measures ANOVA was used (with an intrasubject factor (time) and an intersubject factor (treatment)). Post-hoc corrections to intergroup comparisons were tested by Student's t-test with the Bonferroni correction. For intragroup comparisons, ANOVA with multiple rank test were used to identify differences between successive follow-ups. Statistical analysis was performed with a SPSS software package for Windows (version 17.0; SPSS, Chicago, III), a $p$ value of $<0.05$ was considered as statistically significant.

\section{RESULTS}

Of the twenty-two patients initially enrolled in the present study, twenty completed the nine-month study. Two patients were excluded after failing the first follow-up. No complications such as abscesses or infections were found during the study period, and the interventions were well tolerated and fitted in well with orthodontic treatment.

Both preventive treatments applied in the present work showed reductions in clinical periodontal scores (PI, GI and PD) (Figure 3.A-C), counts of specific periodontopathogens (total bacteria and Pg and Pi) (Figure 4.AE, Table 2) and key inflammatory mediator levels (IL-1 $\beta$, IL-10 and TNF- $\alpha$ ) (Table 3).

The progression of clinical periodontal indices (PI, GI and PD) from baseline through the 3-, 6- and 9-month follow-ups is summarized in Figure 3. PI values showed the same trend in both groups, with a statistically significant decrease $(p<0.05)$ at 3 and 6 months compared to baseline (Figure 3.A). Likewise, GI and PD values (Figures 3.B and 3.C respectively) showed statistically significant decreases in both groups at 3 months compared to baseline. In the PDT group, the decrease in GI and PD values was observed over the whole course of the study period, while in the US group a slight upturn was observed at the 9-month follow-up. Nevertheless, no statistically significant intergroup differences were found for the three clinical parameters analyzed (Figure 3). 
The results of the effect of PDT and US on subgingival microflora during the experimental period are shown in Figure 4.A.E. The reduction in mean total $\log \mathrm{CFU}$ of periodontopathogenic bacteria at each follow-up was more marked in the PDT group, although there were no statistically significant differences in absolute terms between the two treatment modalities at any time point. PDT treatment showed a significant reduction of total $\log$ CFU at 3, 6 and 9 months compared to baseline. With the US treatment however, the difference in reduction of total $\log$ CFU after the three follow-up sessions was not statistically significant (Figure 4.A-E).

Specifically, Table 2 shows the evolution of log CFUs of the three most prevalent periodontopathogens $(P g$, $P i$ and $F n$ ) during this clinical study. Log CFU counts of $P g, P i$ and $F n$ followed similar trends under both treatments tested. A significant reduction in the log CFU of $P i$ and $P g$ was observed in both groups at the 3month follow-up, and to a greater extent in the PDT group ( $p>0.05)$. Between the 3- and 6-month follow-up, the $\log$ CFU count of $P g$ decreased, although a tendency to return to baseline values was observed after 9 months. With respect to $P i$, the log CFU counts remained constant between the third and the ninth month. The $\log$ CFU count for $F n$ in both treatment groups remained constant and statistically significant intragroup differences were not observed. Summing up, in terms of the three periodontopathogen counts, statistically significant differences between the PDT and US treatments were not observed during the experimental period (Table 2).

Table 3 shows the progression in the levels of immune-inflammatory cytokines during the study following US and PDT treatment. In this respect, IL-1 $\beta$ levels in GCF decreased significantly in both treatment groups at the 3-month follow-up, with a further decrease at 6 months, but increased again after 9 months. IL-10 levels in GCF decreased steadily throughout the study period in both treatment groups, with significant reductions $(\mathrm{p}<0.05)$ detected at 3, 6 and 9 months with respect to baseline levels in the PDT group, and at 6 and 9 months compared to baseline in the US group. TNF- $\alpha$ levels in GCF remained stable until 6 months, then decreased significantly at 9 months with respect to 6 months in both treatment groups (Table 3). Both the PDT and US groups displayed stable IL-6 and IL-1ra levels during the experimental period, yielding no statistical significant intra- or intergroup differences. The levels of FGF-2 (FGF basic) increased progressively with respect to baseline after both experimental interventions, particularly in the US group, although there were no statistically significant intra- or intergroup differences (Table 3). 


\section{DISCUSSION}

While there are numerous studies on the effects of PDT as monotherapy or as adjunctive therapy to scaling and root planing (SRP) in the treatment of periodontal disease [20-25], there is only one study on the periodontal effects of PDT in adolescents with fixed orthodontic treatment [13]. Published literature on the effects of PDT or US treatments as nonsurgical periodontal therapies can be used as a support to prudently discuss the results here obtained, taking into account that the selected population did not show clear clinical sings of established gingivitis.

The present clinical study was specifically designed to evaluate and compare the effects of two experimental therapies, PDT and US, on periodontal health (PI, GI, PD), the composition of pathogenic oral microflora, and the secretion levels of target immunological biomarkers in young patients treated with fixed orthodontic appliances. Clinical results showed that both therapies were effective in improving periodontal health outcomes, and even up to 9 months with PDT, which obtained slightly better results than US in terms of decrease of GI and PD. Other authors have likewise remarked on the clinical improvements in periodontal health after PDT treatment versus conventional scaling methods in short population size (twenty patients approx.) affected with chronic periodontitis [20, 21]. In this respect, recent systematic reviews and metaanalyses concluded that use of PDT, whether as adjunctive therapy or unique treatment, was associated with significant improvements in attachment gain after 12 weeks [0.29, 95\% CI 0.08-0.50, p=0.007] and greater reduction in probing depth $[0.11,95 \% \mathrm{CI}:-0.12-0.35, \mathrm{p}=0.35]$ in patients already affected with periodontal disease $[22,23]$. The present study aimed not only to assess the clinical effects, but also to provide further insights into microbiological and protein biomarker responses underlying clinical changes in young patients under orthodontic treatment after PDT or US preventive treatments. On this particular point, although the reduction in total periodontopathogenic flora observed between the groups was not statistically significant, it was more marked in the PDT group, particularly after 3 months. This may correlate with the substantial reduction of GI, along with the decreases in the two specific biomarkers in GCF (IL-1 $\beta$ and IL-10) (Table 3). 
More importantly, it should be pointed out that the patients in the present study were young and free of any form of periodontitis, although they did present some degree of gingival inflammation caused by the orthodontic device. Although the latest generation of ultrasonic scalers have improved patient comfort as well as efficient and effective plaque removal (faster than PDT), in our context, SRP or debridement are not necessary and PDT could represent a slight improvement on conventional methods, due both to its antimicrobial effects and the photobiomodulatory effect of the low power light used to excite the photosensitizer [21, 24, 26]. This last effect could restore cell biological balance improving the vitality conditions of the gingival tissue.

Most of the published literature focusing on the adjunctive use of PDT in periodontal disease has evaluated the effects of a single application of PDT after SRP treatment [21-23, 27, 28]. One example is Oliveira et al, who examined the short-term (3 months) clinical effects in ten patients after a single dose of PDT [28]. The results of the present clinical trial offer new data about the effect of repeated applications of PDT in the short(3 months) and mid-term (6 and 9 months) in a specific sub-cohort of patients. The data at these different timepoints are of critical interest when monitoring gingival health status in an orthodontic population, since gingival heath status is a dynamic phenomenon and represents a constant threat during the entire orthodontic treatment [4]. Available data so far suggest that adjunctive PDT in repeated doses could improve on some of the clinical results observed [29, 30]. Consequently, the present study considered the use of repeated doses for US and PDT, and the results showed a remarkable effect over time deriving from repeated PDT regimens and the application of ultrasonic scaler.

Another sensitive critical point in terms of the resilience of the oral microbiota is the type of photosensitizing agent used in PDT. Methylene blue, which was used in the present clinical trial, is extremely effective for the inactivation of both gram-positive and gram-negative periodontopathogenic species. This is of great importance since the gram-negative microorganisms analyzed here, $P g, P i$ and $F n$, have shown great resistance against PDT compared with gram-positive bacteria because of differences in the outer membrane structures of the two types of bacteria [31]. While preserving the integrity of gingival tissue and preventing secondary gingival lesions [32] this type of photosensitizing agent has also been shown to play an important role in preventing re-colonization of specific periodontopathogens in periodontal lesions [33]. 
The microbiological results of the impact of the two treatments tested in this clinical trial showed that PDT treatment obtained slightly greater reductions in total periodontopathogenic flora. More specifically, substantial reductions in $P g$ (until the 6-month follow-up) and $P i$ (until the 3-month follow-up) were shown following PDT, while Fn remained constant throughout the period of analysis. It is well known that $F n$ can help recruit other pathogenic bacteria, and so contribute subsequently to the initiation and progression of periodontal disease [34]. Positive associations between Fn, Pg, Pi, and Bacteroides forsythus have been reported previously in sub-gingival plaque samples, while $P i$ has never been detected in any site without $F n$ also being present [35]. The results of the present study might indicate that the constant presence of $F n$ during the study favored re-colonization, first by $P i$, then by $P g$. The rate of re-colonization in the PDT group was slower than in the US group. Importantly, with respect to the presence of $P g$, which is considered, with $A a$ [36], to be a critical species in destructive periodontal disease, a significant reduction in the $\log \mathrm{CFU}$ of $P g$ was observed until the 6-month follow-up, correlating with reduced gingival inflammation. Data from the present study showed reductions in both parameters between baseline and 6 months after both PDT and US treatments tested. PDT showed 64.7 and $62.5 \%$ reductions in log CFU of $P g$ and GI, respectively, and US treatment showed 56.1 and $59.6 \%$ reductions in $\log$ CFU of $P g$ and GI, respectively. Trends similar to those found in the present research between $P g$ and GI have been described previously by other authors [37].

The performance effects of conventional ultrasonic debridement versus a single application of PDT in persistent pockets of maintenance patients have been compared previously. The authors reported that microbial counts in both treatment groups returned to baseline values in the short-term (3 months)[20], which reinforces the notion that repeated sessions of PDT are required to achieve proper periodontal health status over a longer period. A significant reduction in pathogenic microbiota reduces pathogenic host immune responses, interferes with the immunostimulatory function of antigen-presenting cells, inactivating proinflammatory mediators and so reducing inflammation and bleeding [38, 39]. Bacterial metabolites stimulate monocytes/macrophages, lymphocytes and other types of cell (fibroblasts and endothelial cells) to secrete cytokines that regulate inflammatory and immune responses. Gram-negative anaerobic rods are the most pathogenic species of the subgingival microflora. Lysis of the outer membrane of Gram-negative bacteria by conventional antimicrobial agents results in the release of the lipopolysaccharide (LPS) inducing local inflammation. Because of the ability of PDT mediated by MB to reduce their key virulence factors such 
as lipopolysaccharide and proteases in the early stages of the immune cascade, its use may, as a result, reduce a number of inflammatory mediators that promote tissue loss associated with periodontal disease [40]. In the case of US, inflammation is reduced by the disruption and physical removal of dental biofilm by cavitation methods that may induce tissue damage of a more invasive nature due to the therapeutic method itself [41]. Differences in the combined microbiome and protein biomarker profiles of individuals with periodontitis and healthy subjects have been described [7, 42]. The present clinical trial also aimed to determine the effects of protein levels, by monitoring a subset array of immunomodulatory mediators $[28,30,38,43]$ in regulating tissue damage and host response, as previously published [44].

In the present study, IL-1 $\beta$ levels in GCF decreased to a greater extent in patients in the PDT group compared to those in the US group. In the same connection, several studies have shown that clinical inflammation decreased in parallel with levels of IL-1 in GCF [45]; more specifically, some authors concluded that in some patients the levels of IL-1 $\beta$ in GCF were higher in sites of gingivitis compared to healthy sites [46]. Significantly increased levels of IL-1 $\beta$ have been shown after de novo mucosal inflammation associated with plaque accumulation in the GCF of associated teeth [47]. Recent evidence has shown similar trends in IL-1 $\beta$ and TNF- $\alpha$ reduction after treating periodontitis lesions with two types of laser radiation [48]. On this point, it has been reported that de novo plaque accumulation does not induce changes in TNF- $\alpha$ levels after a 21-day period of induced gingivitis [47]. Furthermore, in terms of TNF- $\alpha$ secretion levels, the effects of periodontal treatment did not show clear differences between treated vs non-treated patients after 12 months [29, 31, 44]. Coinciding with this scientific evidence, the present study found that TNF- $\alpha$ levels remained stable during the first 6 months after treatment with both therapies, and decreased after 9 months of monitoring, also in both US and PDT groups. Likewise, the values of IL-10 in GCF obtained during the present study decreased during the period of evaluation with both interventions tested. Nevertheless, clear evidence about IL-10 secretion levels has not yet been presented in terms of the presence/absence of gingival inflammation, since IL-10 has been detected in both healthy and inflamed human GCF [49, 50].

Other critical biomarkers of gingival inflammation are IL-1ra and IL-6, whose secretion correlates directly with active inflammation $[51,52]$. The results of the present study showed that both IL-1ra and IL-6 remained stable during the study in both treatment modalities tested in this orthodontic context, while a slight parallel 
increase was observed in levels of FGF-2 (FGF basic) was observed. FGF-2 induces the regeneration of periodontal tissue, including osteogenesis and cementogenesis [53]. Franco et al. showed that PDT in conjunction with conventional dental scaling for periodontal treatment led to increased expression of FGF-2, which favored the regeneration of periodontal tissue [54]. The results of the present work also showed increased levels of FGF-2 (FGF basic) in GCF until the 6-month follow-up in the US group, and the 9-month follow-up in the PDT group.

Clinical studies simultaneously and extensively evaluating the clinical, microbiological and immunological impact of PDT and other preventive methods in the treatment of gingivitis during orthodontic treatment are still very scarce. Our study presents for the first time useful comparative clinical, microbiological and protein marker data, recording the effects of two well-described therapies in the context of periodontal health maintenance on a subset of strictly controlled orthodontic patients. Although both two treatments resulted in clinical, microbiological and immunological improvement, the clear superiority of one procedure over the other could not be demonstrated. In the context of maintenance care, both procedures are well tolerated with no side effects even when repeated multiple times. Consequently, both procedures have therapeutic potential.

\section{CONCLUSIONS}

The present clinical trial led to the conclusion that both photodynamic therapy and ultrasonic scaling are safe and effective treatment methods for gingival inflammation induced by fixed orthodontic appliances. In terms of clinical, microbiological and anti-inflammatory outcomes, PDT was slightly more effective than the implementation of the US, allowing the extension of the benefits for a longer period. PDT could therefore be consolidated as a new safe therapeutic alternative for supportive periodontal maintenance routines in patients treated with orthodontic fixed appliances. 


\section{REFERENCES}

[1] Verrusio C, Iorio-Siciliano V, Blasi A, Leuci S, Adamo D, Nicolò M. The effect of orthodontic treatment on periodontal tissue inflammation: A systematic review. Quintessence Int 2018; 49 (1): 69-77.

[2] Mártha K, Mezei T, János K. A histological analysis of gingival condition associated with orthodontic treatment. Rom J Morphol Embryol 2013; 54 (3 suppl): 823-827.

[3] Su Y, Chen C, Guo L, Du J, Li X, Liu Y. Ecological balance of oral microbiota is required to maintain oral mesenchymal stem cell homeostasis. Stem Cell 2017; 36(4): 551-561.

[4] Antoun JS, Mei L, Gibbs K, Farella M. Effect of orthodontic treatment on the periodontal tissues. Periodontol 2000 2017; 74(1): 140-157.

[5] Martignon S, Ekstrand K, Lemos M, Lozano M, Higuera C. Plaque, caries level and oral hygiene habits in young patients receiving orthodontic treatment. Comm Dent Health 2010; 27(3): 133-138.

[6] Krishnan V, Ambili R, Davidovitch Z, Murphy N.C. Gingiva and orthodontic treatment. Semin Orthod 2007; 13:257-271.

[7] Rosier BT, Marsh PD, Mira A. Resilience of the oral microbiota in health: mechanisms that prevent dysbiosis. J Dent Res 2018; 97(4): 371-380.

[8] Schwarz F, Aoki A, Becker J, Sculean A. Laser application in non-surgical periodontal therapy: a systematic review. J Clin Periodontol 2008; 35 (s8): 29-44.

[9] Yin R, Hamblin MR. Antimicrobial photosensitizers: drug discovery under the spotlight. Curr Med Chem 2015; 22 (18): 2159-2185. 
[10] Pfitzner A, Sigusch BW, Albrecht V, Glockmann E. Killing of periodontopathogenic bacteria by photodynamic therapy. J Periodontol 2004; 75(10): 1343-1349.

[11] Konopka K, Goslinski T. Photodynamic therapy in dentistry. J Dent Res 2007; 86(8): 694-707.

[12] Jori G, Fabris C, Soncin M, et al. Photodynamic therapy in the treatment of microbial infections: basic principles and perspective applications. Lasers Surg Med 2006; 38(5): 468-481.

[13] Paschoal MA, Moura CM, Jeremias F, et al. Longitudinal effect of curcumin-photodynamic antimicrobial chemotherapy in adolescents during fixed orthodontic treatment: a single-blind randomized clinical trial study. Lasers Med Sci 2015; 30(8): 2059-2065.

[14] Panhóca VH, Esteban Florez FL, Corrêa TQ, Paolillo FR, de Souza CW, Bagnato VS. Oral Decontamination of Orthodontic Patients Using Photodynamic Therapy Mediated by Blue-Light Irradiation and Curcumin Associated with Sodium Dodecyl Sulfate. Photomed Laser Surg 2016; 34(9): 411-417.

[15] World Medical Association Declaration of Helsinki: ethical principles for medical research involving human subjects- World Medical Association. JAMA 2013; 310(20): 2191-2194.

[16] Silness J, Loe H. Periodontal disease in pregnancy. II. Correlation between oral hygiene and periodontal condition. Acta Odontol Scand 1964; 22: 121-135.

[17] Loe H. The gingival index, the plaque index and the retention index systems. J Periodontol 1967; 38(6) Suppl: 610-616.

[18] Alsina M. Olle E, Frias J. Improved, low-cost selective culture medium for Actinobacillus actinomycetemcomitans. J Clin Microbiol 2001; 39(2): 509-513.

[19] Chapple ILC, Landini G, Griffiths GS, Patel NC, Ward RSN. Calibration of the Periotron $8000^{\circledR}$ and $6000^{\circledR}$ by polynomial regression. J Periodont Res $1999 ; 34: 79-86$.

[20] Rühling A, Fanghänel J, Houshmand $M$, et al. Photodynamic therapy of persistent pockets in maintenance patients - A clinical study. Clin Oral Investig 2010; 14(6): 637-644.

[21] Braun A, Dehn C, Krause F, Jepsen S. Short-term clinical effects of adjunctive antimicrobial photodynamic therapy in periodontal treatment: a randomized clinical trial. J Clin Periodontol 2008; 35(10): 877-884.

[22] Atieh MA. Photodynamic therapy as an adjunctive treatment for chronic periodontitis: A meta-analysis. Lasers Med Sci 2010; 25: 605-613.

[23] Sgolastra F, Petrucci A, Gatto R, Marzo G, Monaco A. Photodynamic therapy in the treatment of chronic periodontitis: a systematic review and meta-analysis. Lasers Med Sci 2013; 28(2): 669-682.

[24] Lui J, Corbet EF, Jin L. Combined photodynamic and low-level laser therapies as an adjunct to nonsurgical treatment of chronic periodontitis. J Periodontal Res 2011; 46: 89-96.

[25] Borekci T, Meseli SE, Noyan U, Kuru BE, Kuru L. Efficacy of adjunctive photodynamic therapy in the treatment of generalized aggressive periodontitis: A randomized controlled clinical trial. Lasers Surg Med 2018; doi:10.1002/1sm.23010.

[26] Calderín S, García-Núñez JA, Gómez C. Short-term clinical and osteoimmunological effects of scaling and root planing complemented by simple or repeated laser phototherapy in chronic periodontitis. Lasers Med Sci 2013; 28: 157-166.

[27] Polansky R, Haas M, Hesch A, Wimmer G. Clinical effectiveness of photodynamic therapy in the treatment of periodontitis. J Clin Periodontol 2009; 36(7): 575-580. 
[28] de Oliveira RR, Schwartz-Filho HO, Novaes Jr AB, et al. Antimicrobial Photodynamic Therapy in the Non-Surgical Treatment of Aggressive Periodontitis: Cytokine Profile in Gingival Crevicular Fluid, Preliminary Results. J Periodontol 2009; 80(1): 98-105.

[29] Lulic M, Leiggener Görög I, Salvi GE, Ramseier CA, Mattheos N, Lang NP. One-year outcomes of repeated adjunctive photodynamic therapy during periodontal maintenance: a proof-of-principle randomizedcontrolled clinical trial. J Clin Periodontol 2009; 36(8): 661-666.

[30] Moreira AL, Novaes Jr, AB, Grisi MF, et al. Antimicrobial photodynamic therapy as an adjunct to nonsurgical treatment of aggressive periodontitis: a split-mouth randomized controlled trial. J Periodontol 2015; 86(3): 376-386.

[31] Takasaki AA, Aoki A, Mizutani K, et al. Application of antimicrobial photodynamic therapy in periodontal and peri-implant diseases. Periodontol 2000 2009; 51: 109-140.

[32] Malgikar S, Reddy SH, Sagar SV, Satyanarayana D, Reddy GV, Josephin JJ. Clinical effects of photodynamic and low-level laser therapies as an adjunct to scaling and root planing of chronic periodontitis: A split-mouth randomized controlled clinical trial. Indian J Dent Res 2016; 27(2): 121-126.

[33] Chan Y, Lai CH. Bactericidal effects of different laser wavelengths on periodontopathic germs in photodynamic therapy. Lasers Med Sci 2003; 18: 51-55.

[34] Kolenbrander PE. Oral microbial communities: biofilms, interactions, and genetic systems. Annu Rev Microbiol 2000; 54: 413-437.

[35] Signat B, Roques C, Poulet P, Duffaut D. Fusobacterium nucleatum in periodontal health and disease. Curr Issues Mol Biol 2011; 13(2): 25-36.

[36] Slots J, Ting M. Actinobacillus actinomycetemcomitans and Porphyromonas gingivalis in human periodontal disease: occurrence and treatment. Periodontol 2000 1999; 20: 82-121.

[37] Pan S, Liu Y, Si Y, et al. Prevalence of fimA genotypes of Porphyromonas gingivalis on adolescent orthodontic patients. PLoS One 2017; 12(11): e0188420.

[38] de Oliveira RR, Novaes Jr AB, Garlet GP, et al. The effect of a single episode of antimicrobial photodynamic therapy in the treatment of experimental periodontitis. Microbiological profile and cytokine pattern in the dog mandible. Lasers Med Sci 2011; 26(3): 359-367.

[39] Séguier S, Souza SL, Sverzut AC, et al. Impact of photodynamic therapy on inflammatory cells during human chronic periodontitis. J Photochem Photobiol B 2010; 101 (3): 348-354.

[40] Kömerik N, Wilson M, Poole S. The effect of photodynamic action on two virulence factors of Gramnegative bacteria. Photochem Photobiol 2000; 72 (5): 676-680.

[41] Vyas N, Pecheva E, Dehghani H, et al. High Speed Imaging of Cavitation around Dental Ultrasonic Scaler Tips. PLoS One 2016; 11(3): e0149804.

[42] Zhou J, Yao Y, Jiao K, et al. Relationship between Gingival Crevicular Fluid Microbiota and Cytokine Profile in Periodontal Host Homeostasis. Front Microbiol 2017; 8: 2144.

[43] Koçak E, Sağlam M, Kayış SA, et al. Nonsurgical periodontal therapy with/without diode laser modulates metabolic control of type 2 diabetics with periodontitis: a randomized clinical trial. Lasers Med Sci 2016; 31(2): 343-353.

[44] da Cruz Andrade PV, Euzebio Alves T, de Carvalho VF, et al. Photodynamic therapy decrease immuneinflammatory mediators levels during periodontal maintenance. Lasers Med Sci 2017; 32(1): 9-17. 
[45] Sanglam M, Kantarci A, Dundar N, Hakki SS. Clinical and biochemical effects of diode laser as an adjunct to nonsurgical treatment of chronic periodontitis: a randomized controlled clinical trial. Lasers Med Sci 2014; 29(1): 37-46.

[46] Boronat-Catalá M, Catalá-Pizarro M, Bagán Sebastián JV. Salivary and crevicular fluid interleukins in gingivitis. J Clin Exp Dent 2014; 6(2): e175-e179.

[47] Schierano G, Pejrone G, Brusco P. et al. TNF- $\alpha$, TGF- $\beta 2$ and IL-1 $\beta$ levels in gingival and peri-implant fluid before and after de novo plaque accumulation. J Clin Periodontol 2008; 35(6): 532-538.

[48] Sağlam M, Köseoğlu S, Taşdemir I, Erbak Yilmaz H, Savran L, Sütçü R. Combined application of Er:YAG and Nd:YAG lasers in treatment of chronic periodontitis. A split-mouth, single-blind, randomized controlled trial. J Periodontal Res 2017; 52(5): 853-862.

[49] Johannsen A, Bjurshammar N, Gustafsson A. The influence of academic stress on gingival inflammation. Int J Dent Hyg 2010; 8(1): 22-27.

[50] Hirose M, Ishihara K, Saito A, Nakagawa T, Yamada S, Okuda K. Expression of cytokines and inducible nitric oxide synthase in inflamed gingival tissue. J Periodontol 2001; 72(5): 590-597.

[51] Boström L, Linder LE, Bergström J. Smoking and GCF levels of IL-1beta and IL-1ra in periodontal disease. J Clin Periodontol 2000; 27(4): 250-255.

[52] Yucel-Lindberg T, Båge T. Inflammatory mediators in the pathogenesis of periodontitis. Expert Rev Mol Med 2013; 15: e7.

[53] Murakami S. Periodontal tissue regeneration by signaling molecule(s): what role does basic fibroblast growth factor (FGF-2) have in periodontal therapy? Periodontol 2000 2011; 56(1): 188-208.

[54] Franco EJ, Pogue RE, Sakamoto LH, Cavalcante LL, Carvallo, DR, Andrade RV. Increased expression of genes after periodontal treatment with photodynamic therapy. Photodiagn Photodyn Ther 2014; 11(1): 4147.

[55] Socransky SS, Haffajee AD, Cugini MA, Smith C, Kent RL. Jr. Microbial complexes in subgingival plaque. J Clin Periodontol 1998; 25:134-144. 


\section{Figure captions}

Figure 1.- Graphical representation of the clinical trial timeline and the timepoints used for experimental interventions and clinical, microbiological and protein marker assessment.

Figure 2.- A) Application of methylene blue solution into the pocket by a Dentsply 23 gauge blunt ended side-port irrigating needle. B) Photoactivation by a diode laser ended in a disposable light-diffusing tip (configured similar to a periodontal probe), covering a stainless steel autoclavable handpiece. C) Disruption of dental biofilm by US.

Figure 3.- Representative graph showing clinical parameter monitoring at start and at 3,6 and 9 months in the US and PDT groups. A) plaque index (PI) monitoring; B) gingival index (GI) monitoring; C) probing depth (PD) monitoring. Mean Scores $( \pm \mathrm{SD}),(\mathrm{n}=10$ patients/group); $(*)$ Significant intragroup differences at 3month follow-up with respect to baseline; $(\dagger)$ Significant intragroup differences at 6-month follow-up with respect to baseline; $(\wedge)$ Significant intragroup differences at 9-month follow-up with respect to 6-month follow-up. No statistically significant differences were detected for any of the clinical outcome measurements between PDT and US groups at any of the evaluation times.

Figure 4.- Graphical representation of oral microbiota progression in both experimental groups. A) Decimal logarithm-transformed colony-forming units ( $\log \mathrm{CFU}$ ) of total periodontopathogenic flora. Mean Scores $( \pm \mathrm{SD}),\left(\mathrm{n}=10\right.$ patients/group) at baseline, and at 3-, 6-, and 9-month follow-ups; $\left(^{*}\right)$ Significance of intragroup differences compared to baseline at 3-month follow-up; ( $\dagger$ ) Significance of intragroup differences compared to baseline at 6-month follow-up; $\left({ }^{\circ}\right)$ Significance of intragroup differences compared to baseline at 9-month follow-up. No statistically significant differences were detected for any bacteria counts between PDT and US groups at any of the evaluation times. B) Distribution of microbiological complexes within both treatment groups, PDT and US, at baseline, and at 3-, 6-, and 9-month follow-ups. The color represents the different complexes described by Socransky et al., $(1998)^{55}$. RC0: none of the pathogens of the 'red complex' present; RC1: one of the pathogens of the 'red complex' present; RC2: two of the pathogens of the 'red complex' present. 
Table 1. Summary of PDT and US protocol used in this study.

\section{PDT}

PS characteristics: solution of MB $0.005 \% \mathrm{w} / \mathrm{v}$

PS incubation time: $3 \mathrm{~min}$

Photoexcitation wavelength (nm): $670 \mathrm{~nm}$

Irradiation mode: continuous mode

Power output (mW): 190

Irradiance $\left(\mathrm{W} / \mathrm{cm}^{2}\right): 6.05$

Estimated average fluence $\left(\mathrm{J} / \mathrm{cm}^{2}\right): 67.06$

Time of light exposure: $60 \mathrm{sec} ; 30 \mathrm{sec} / \mathrm{buccal}$ tooth surface; $30 \mathrm{sec} /$ lingual tooth surface

\section{US}

Power setting $3(240 \mu \mathrm{m} ; 6000 \mathrm{~Hz} ; 71 \mathrm{~dB}(\mathrm{~A}))$

Contact accessory tip: SONICflex scaler no.5

Application from coronal to apical direction in sweeping motion

Ps: Photosensitizer (Methylene blue, MB) 
Table 2. Colony-forming units ( $\log 10 \mathrm{CFU} / \mathrm{mL})$ of Porphyromonas gingivalis, Prevotella intermedia and Fusobacterium nucleatum: at baseline and at 3-, 6- and 9-month follow-ups.

\begin{tabular}{|c|c|c|c|c|c|}
\hline $\begin{array}{l}\text { Periodonto- } \\
\text { pathogens }^{\delta}\end{array}$ & $\begin{array}{l}\text { Treatment } \\
\text { type }\end{array}$ & $\begin{array}{l}\text { Baseline } \\
\text { Mean } \pm \text { SD }\end{array}$ & $\begin{array}{l}3 \text { months } \\
\text { Mean } \pm \text { SD }\end{array}$ & $\begin{array}{l}6 \text { months } \\
\text { Mean } \pm \text { SD }\end{array}$ & $\begin{array}{l}9 \text { months } \\
\text { Mean } \pm \text { SD }\end{array}$ \\
\hline \multirow[t]{2}{*}{$P g$} & PDT & $4.73 \pm 1.91$ & $2.32 \pm 2.30 *$ & $1.67 \pm 1.60$ & $3.10 \pm 2.18^{\wedge}$ \\
\hline & US & $4.56 \pm 0.58$ & $1.93 \pm 1.90 *$ & $2.00 \pm 1.95$ & $3.89 \pm 2.15^{\wedge}$ \\
\hline \multirow[t]{2}{*}{$P i$} & PDT & $4.32 \pm 1.02$ & $2.60 \pm 2.26^{*}$ & $2.73 \pm 2.07$ & $3.02 \pm 2.11$ \\
\hline & US & $4.37 \pm 1.72$ & $2.69 \pm 1.99 *$ & $2.61 \pm 1.91$ & $3.38 \pm 1.72$ \\
\hline
\end{tabular}

Pg: Porphyromonas gingivalis; Pi: Prevotella intermedia; Fn: Fusobacterium nucleatum; PDT: Photodynamic therapy; US: Ultrasonic scaler; SD: standard deviation; *: Significant difference from baseline to 3 months $(p<0.05) ; \wedge$ : Significant difference from 6 months to 9 months $(p<0.05)$. No statistically significant differences were detected for any bacteria counts between PDT and US groups at any of the evaluation times. ${ }^{\delta}$ Most prevalent periodontopathogens selected from the nine analyzed species. Aa was not detected in any of the patients included in the RCT. 
Table 3. Levels of Interleukin 1 beta, Interleukin 10, Tumor Necrosis Factor Alpha, Interleukin 6, Interleukin-1 Receptor Antagonist and Fibroblast Growth Factor 2 in GCF at baseline and at 3-, 6- and 9month follow-ups.

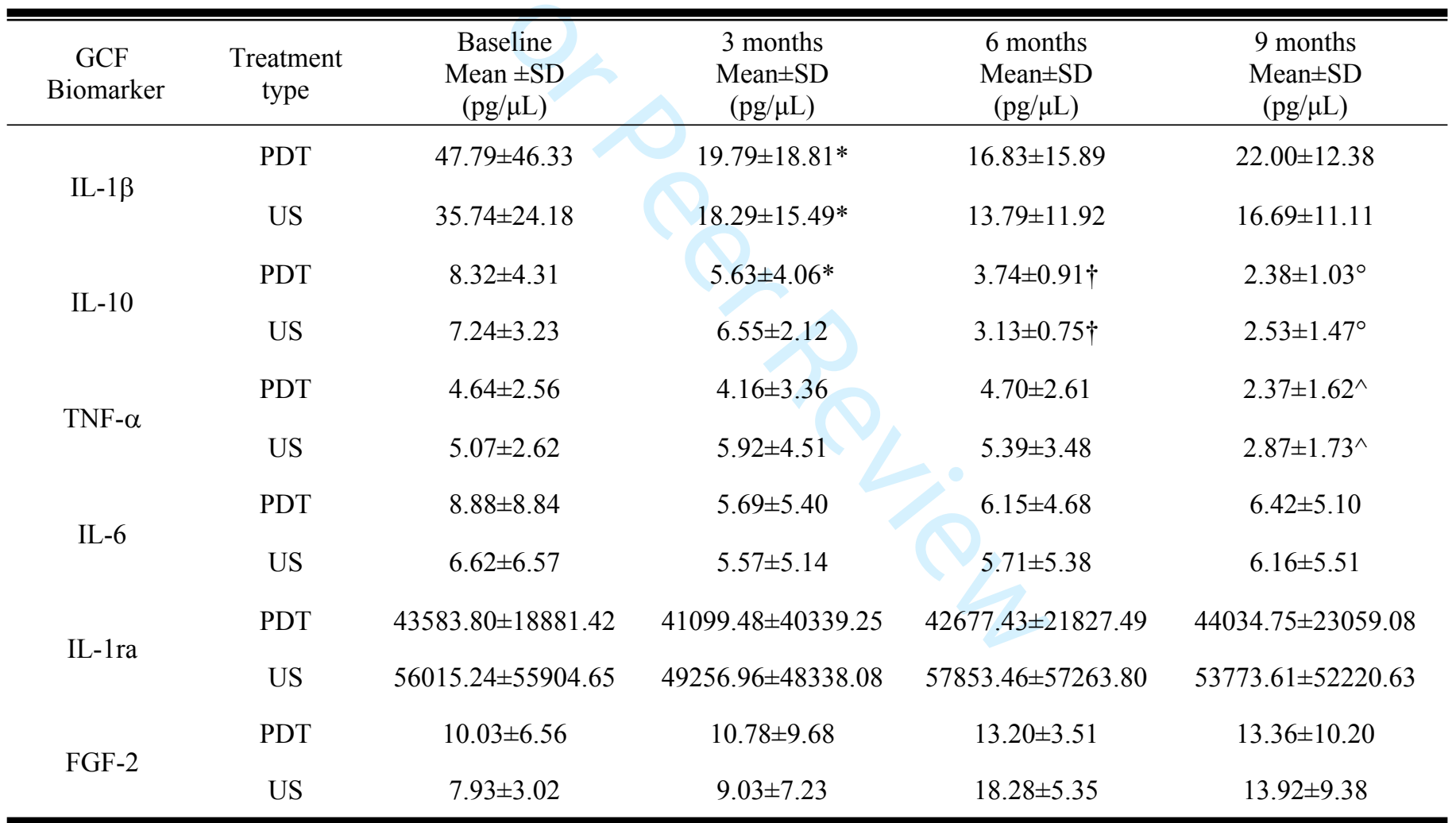

GCF: Gingival crevicular fluid; PDT: Photodynamic therapy; US: Ultrasonic scaler; IL-1ß: Interleukin 1beta; IL10: Interleukin 10; TNF- $\alpha$ : Tumor Necrosis Factor Alpha; IL-6: Interleukin 6; IL-1ra: Interleukin-1 Receptor Antagonist; FGF-2: Fibroblast Growth Factor 2; SD: standard deviation; *: Significant difference from baseline to 3 months $(p<0.05)$; ${ }^{\dagger}$ : Significant difference from baseline to 6 months $(p<0.05) ;{ }^{\circ}$ : Significant difference from baseline to 9 months $(p<0.05)$; : Significant difference from 6 months to 9 months $(p<0.05)$. No statistically significant differences were detected for any protein marker levels between PDT and US groups at any evaluation times. 
Figure 1.- Graphical representation of the clinical trial timeline and the timepoints used for experimental interventions and clinical, microbiological and protein marker assessment.

$120 \times 87 \mathrm{~mm}(300 \times 300$ DPI $)$ 
A

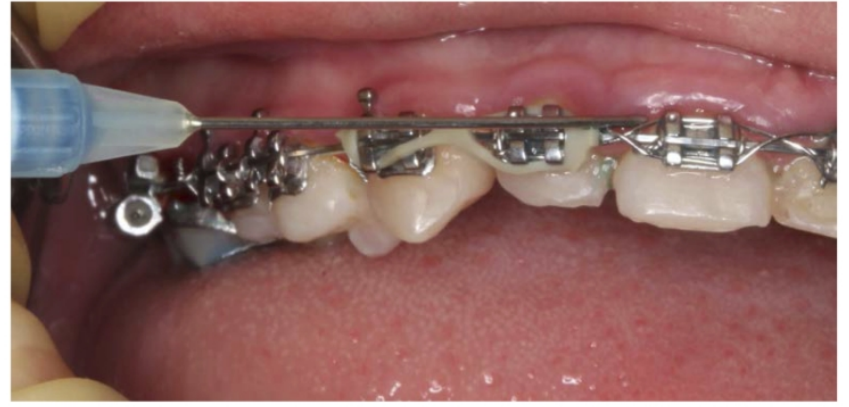

B

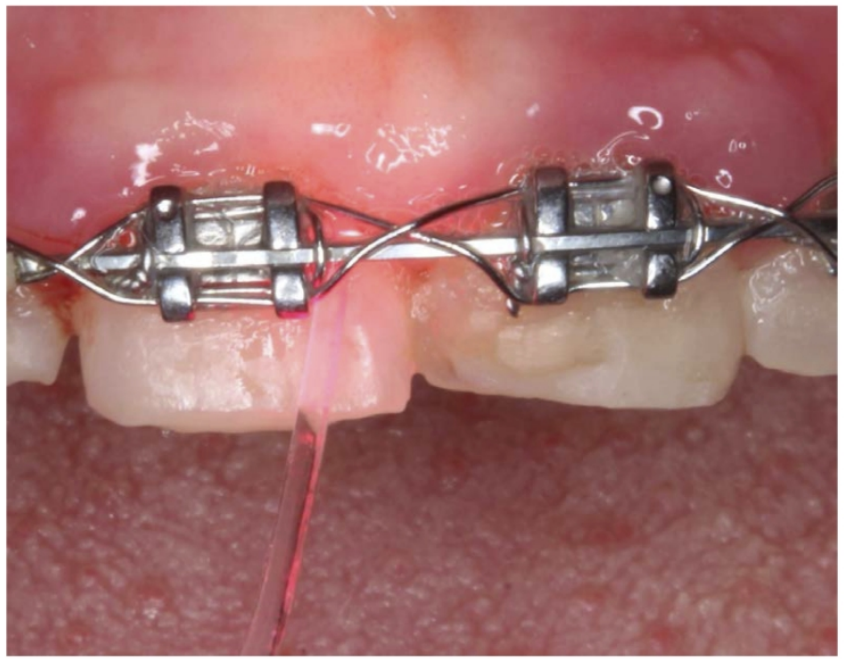

C

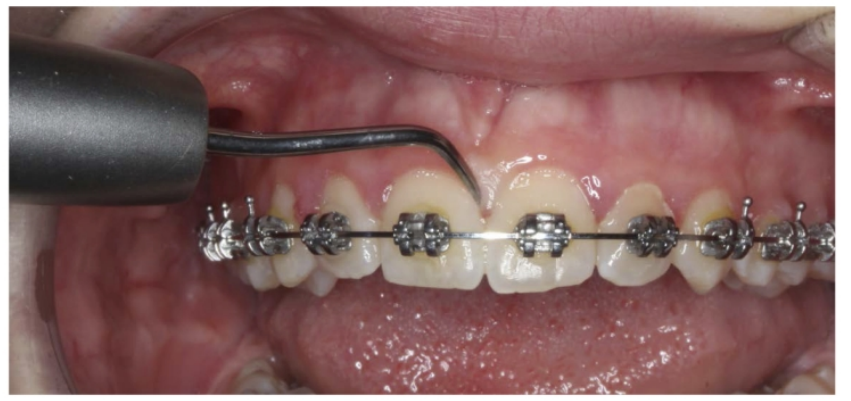

Figure 2

Figure 2.- A) Application of methylene blue solution into the pocket by a Dentsply 23 gauge blunt ended side-port irrigating needle. B) Photoactivation by a diode laser ended in a disposable light-diffusing tip (configured similar to a periodontal probe), covering a stainless steel autoclavable handpiece. C) Disruption of dental biofilm by US.

$175 \times 249 \mathrm{~mm}(300 \times 300 \mathrm{DPI})$ 

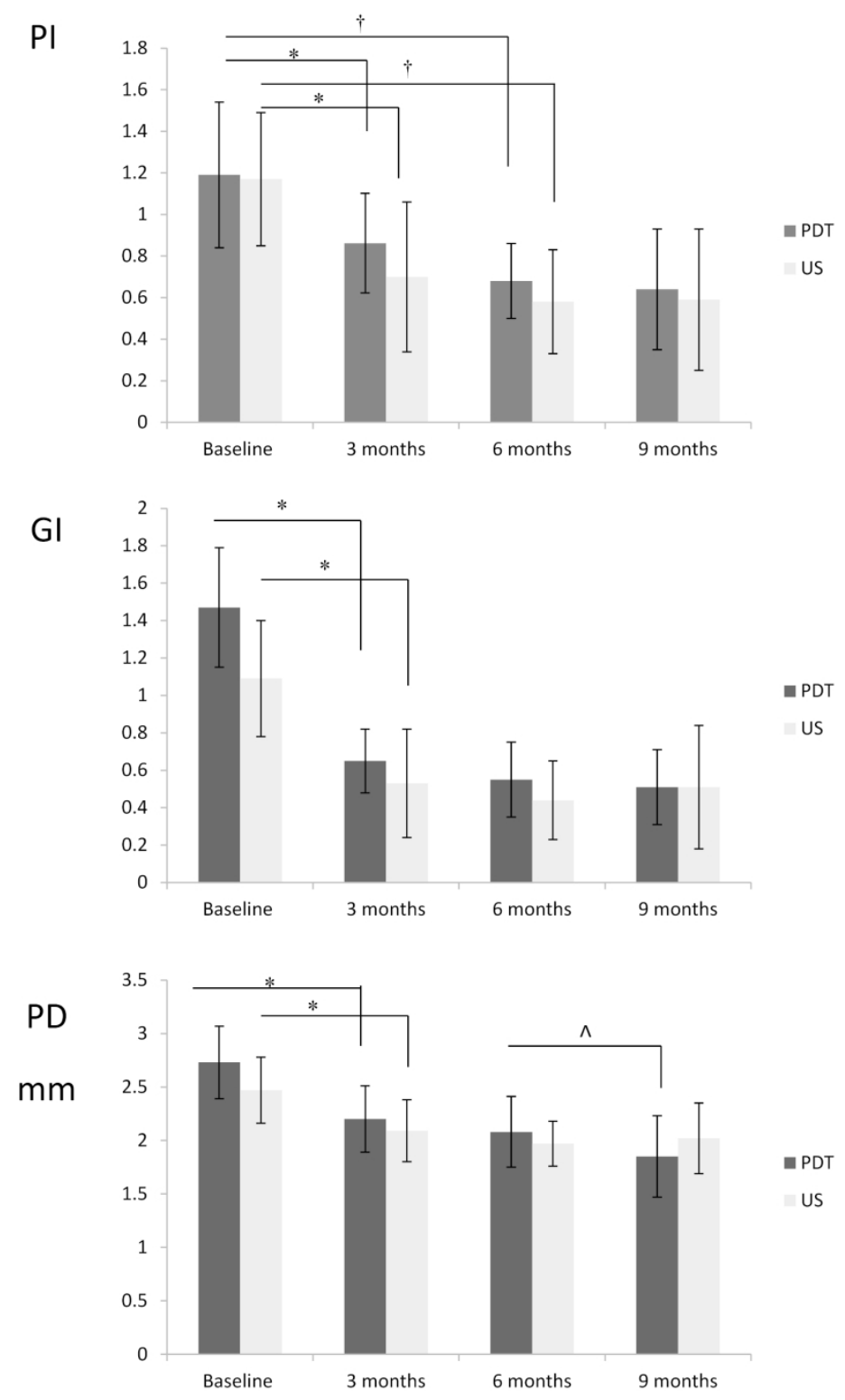

Figure 3

Figure 3.- Representative graph showing clinical parameter monitoring at start and at 3,6 and 9 months in the US and PDT groups. A) plaque index (PI) monitoring; B) gingival index (GI) monitoring; C) probing depth (PD) monitoring. Mean Scores $( \pm S D),(n=10$ patients/group); $(*)$ Significant intragroup differences at 3-month follow-up with respect to baseline; ${ }^{\dagger}+$ Significant intragroup differences at 6-month follow-up with respect to baseline; ( $\left.{ }^{\wedge}\right)$ Significant intragroup differences at 9-month follow-up with respect to 6-month follow-up. No statistically significant differences were detected for any of the clinical outcome measurements between PDT and US groups at any of the evaluation times.

$$
171 \times 239 \mathrm{~mm} \text { ( } 300 \times 300 \text { DPI) }
$$




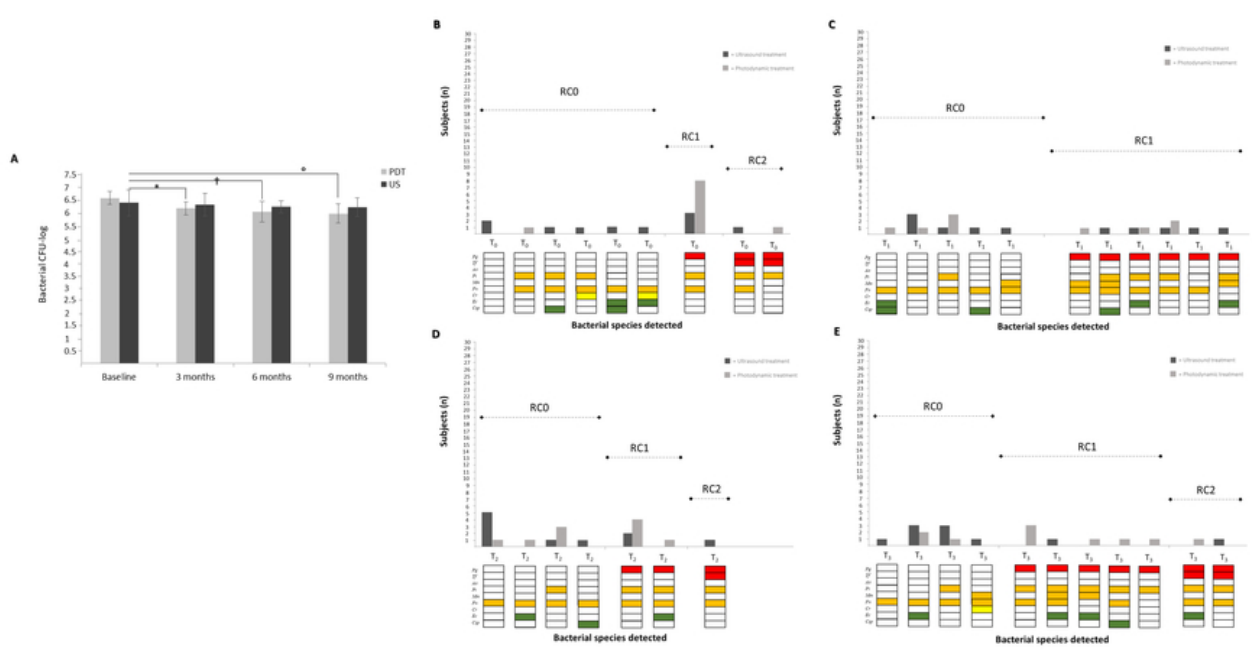

Figure 4.- Graphical representation of oral microbiota progression in both experimental groups. A) Decimal logarithm-transformed colony-forming units (log CFU) of total periodontopathogenic flora. Mean Scores $( \pm \mathrm{SD}),(\mathrm{n}=10$ patients/group) at baseline, and at 3-, 6-, and 9-month follow-ups; (*) Significance of intragroup differences compared to baseline at 3-month follow-up; $\left.{ }^{\dagger}\right)$ Significance of intragroup differences compared to baseline at 6-month follow-up; $\left(^{\circ}\right)$ Significance of intragroup differences compared to baseline at 9-month follow-up. No statistically significant differences were detected for any bacteria counts between PDT and US groups at any of the evaluation times. B) Distribution of microbiological complexes within both treatment groups, PDT and US, at baseline, and at 3-, 6-, and 9-month follow-ups. The color represents the different complexes described by Socransky et al., (1998) [55]. RC0: none of the pathogens of the 'red complex' present; RC1: one of the pathogens of the 'red complex' present; RC2: two of the pathogens of the 'red complex' present.

$76 \times 38 \mathrm{~mm}(300 \times 300 \mathrm{DPI})$ 